\title{
Incidence of Diabetic Nephropathy and Its Predictors among Type 2 Diabetes Mellitus Patients at University of Gondar Comprehensive Specialized Hospital, Northwest Ethiopia
}

\author{
Sewnet Adem Kebede $\mathbb{D}^{1},{ }^{1}$ Biruk Shalmeno Tusa ${ }^{\mathbb{D}},{ }^{2}$ Adisu Birhanu Weldesenbet $\mathbb{D}{ }^{2}$ \\ Zemenu Tadesse Tessema $\oplus^{1},{ }^{1}$ and Tadesse Awoke Ayele $\mathbb{C}^{1}$ \\ ${ }^{1}$ Department of Epidemiology and Biostatistics, Institute of Public Health, College of Medicine and Health Sciences, \\ University of Gondar, Gondar, Ethiopia \\ ${ }^{2}$ Department of Epidemiology and Biostatistics, Collage of Health and Medical Sciences, Haramaya University, \\ Haramaya, Ethiopia
}

Correspondence should be addressed to Sewnet Adem Kebede; sewnetme1@gmail.com

Received 5 June 2021; Accepted 20 August 2021; Published 29 August 2021

Academic Editor: C. S. Johnston

Copyright (C) 2021 Sewnet Adem Kebede et al. This is an open access article distributed under the Creative Commons Attribution License, which permits unrestricted use, distribution, and reproduction in any medium, provided the original work is properly cited.

\begin{abstract}
Background. Although the rate of diabetic nephropathy which is the leading cause of end-stage renal disease (ESRD) continues to rise, there is limited information about the problem. This study aimed to assess the incidence and predictors of diabetic nephropathy among type $2 \mathrm{DM}$ patients. Methods. Institution-based retrospective follow-up study was conducted at UGCSH with 462 newly diagnosed type 2 DM patients from January 2001 to February 2016, and the data were collected by reviewing their records. The Schoenfeld residuals test was used to check proportional hazard assumption. The best model was selected by using Akaike information criteria (AIC). Hazard ratios (HR) with its respective 95\% confidence interval were reported to show significance and strength of association. Results. The incidence rate of diabetic nephropathy was 14 (95\% CI 10.8-17.7) cases per 10,000 patient-month observation. In addition, 63 (13.6\%) DM patients developed diabetic nephropathy. The median time to develop diabetic nephropathy was 94.9 months with interquartile range (IOR) of (64.1-127.4) months. Type 2 DM patients who had coronary heart disease $(\mathrm{AHR}=2.69,95 \% \mathrm{CI} 1.42-5.13)$ and anemia $(\mathrm{AHR}=1.94,95 \% \mathrm{CI} 0.97-3.87)$ were at higher hazard for developing diabetic nephropathy. Besides this, having a long duration ( $>10$ years) (AHR $=0.24,95 \%$ CI $0.11-0.56)$ and being female ( $\mathrm{AHR}=0.44,95 \%$ CI 0.26-0.73) was found to be protective against diabetic nephropathy. Conclusion. The incidence of diabetic nephropathy among type 2 diabetes patients remains a significant public health problem. Duration of diabetes $>10$ years and female sex reduced the risk of diabetic nephropathy. Coronary heart disease and anemia increased the risk of diabetic nephropathy among type $2 \mathrm{DM}$ patients. In light of these findings, early screening for diabetes complication is needed, and health professionals should give targeted intervention for type $2 \mathrm{DM}$ patients with coronary heart disease comorbidity and anemia.
\end{abstract}

\section{Introduction}

Diabetic nephropathy (DN) is one of the most common microvascular complications of diabetes and a leading cause of morbidity and mortality in diabetic patients $[1,2]$. This condition is a result of vascular abnormalities that accompany diabetes and increases mortality risk [3]. It is also the leading cause of end-stage renal disease (ESRD) worldwide and a leading cause of DM-related morbidity and mortality
$[4,5]$. The proportion of ESRD attributable to diabetes alone ranges from $12 \%$ to $55 \%$ [1].

The rise in DN prevalence corresponds to the dramatic rise in diabetes prevalence around the world. Approximately 463 million adults aged $20-79$ years are currently living with diabetes. Almost half (46.2\%) of deaths associated with diabetes occur in people under the age of 60 years [6]. Due to the effect of globalization and epidemiologic transition, it is estimated that $79.4 \%$ of adults with diabetes live in low- and 
middle-income countries [7, 8]. In the United Kingdom, $25 \%$ of people with diabetes and in the United States of America, 36\% of people with diabetes have diabetic nephropathy [6]. The prevalence of $\mathrm{DN}$ in Africa varied from $11 \%$ to $83.7 \%$ [9].

The pathogenesis of $\mathrm{DN}$ is complex and multifactorial. According to different literatures, the most common risk factors for development of diabetic nephropathy complication among type 2 diabetic patients include sex, age, body mass index, hypertension, duration, and fasting blood sugar [10-12]. Studies also have illustrated a tight relationship between diabetic nephropathy and diabetic retinopathy [13-15]. However, the importance of the above factors varies between studies.

The increasing prevalence of the diabetes mellitus is linked with the emergence of diabetes complication as a cause of premature death and disability. It is also associated with a negative economic impact for many countries $[6,16,17]$. People with diabetes and clinical nephropathy experience $50 \%$ higher health expenditures compared to those with diabetes but without clinical nephropathy [18]. Sustainable development goal three of the United Nations has targeted to reduce diabetes and its severity among other noncommunicable diseases such as cardiovascular diseases, cancers, and chronic respiratory diseases [19].

Several studies in Ethiopia have shown that the presence and severity of complications related to diabetic nephropathy like ESRD are steadily increasing, and these are the causes of premature death, disability, and negative economic impact $[6,16-18]$. In developed countries, the incidence and risk factors of diabetic nephropathy have been well documented. However, studies regarding the incidence of diabetic nephropathy and its predictors are scarce in Ethiopia. To present, most of epidemiological research on diabetic nephropathy in Africa including Ethiopia has been limited to prevalence estimation from cross-sectional studies. Estimating the incidence of DN and early detection of the risk factors is important for the prevention of $\mathrm{DN}$. Thus, we determined the incidence and predictors of diabetic nephropathy among type 2 diabetes patients.

\section{Materials and Methods}

2.1. Study Area. The study was conducted at University of Gondar Comprehensive Specialized Hospital, which is a teaching hospital. The hospital serves for greater than 5 million people in northwest Ethiopia. Around 24,862 numbers of people are having chronic follow-up per year, and among this, 8,900 are DM patients.

2.2. Study Design and Subjects. An institutional-based retrospective follow-up study was conducted. All newly diagnosed type 2 diabetes mellitus (T2DM) patients who are enrolled from January 2001 to February 2016 at University of Gondar Comprehensive Specialized Hospital were considered in this study. New T2DM diagnosed patients were eligible, while those who had diabetic nephropathy at the time of the diagnosis for T2DM were excluded from the study.
2.3. Sample Size and Sampling Technique. The required sample size was calculated via Stata software using power analysis for the log rank test by considering the following assumptions: survival probability of those having $\mathrm{HDL}$ $<40 \mathrm{mg} / \mathrm{dl}(p=0.77)$ [10], since the HDL level is an independent risk factor for the development of microvascular disease affecting the kidney in patients with type 2 diabetes [20], 95\% confidence level, and 5\% margin of error. Therefore, the total calculated sample size was 462 .

2.4. Data Collection Methods. The study used secondary data; a data extraction check list was prepared to collect the data. The reviewed records were identified by their medical registration number. Patient intake form follow-up card and DM registration book are used as data sources. Sociodemographic characteristics, baseline, and follow-up clinical and laboratory data were collected from patient cards. The date that patients start regular follow-up treatment until the end of the study to the confirmation of a final event in the study period was collected.

2.5. Data Quality Control Methods. A week before the actual data collection, preliminary review was done on similar area. The data extraction sheet was the pretest for consistency of understanding, adequacy of instrument, time requirement to fill the check list of tools, and completeness of data for charts. Necessary adjustment for the final data collection sheet was made by excluding variables which are not found on charts. Training on the objective of the study and how to retrieve records as per data extraction sheet was given to data collectors and supervisors two days before data collection. As well as, random sample from data extracted was crosschecked for its consistency. The information formats were crosschecked with the source card on the spot, and regular supervision was done.

2.6. Data Processing and Analysis. After they were checked for completeness, data were entered using Epi Info 7 and exported to STATA 14 for further analysis. The outcome variable in this study was time to diabetic nephropathy. Diabetic nephropathy was defined as an estimated glomerular filtration rate $(\mathrm{eGFR})<60 \mathrm{ml} / \mathrm{min} / 1.73 \mathrm{~m}^{2}$ estimated by the Cockcroft-Gault equation. [21, 22] Accordingly, participants were classified as either diabetic nephropathy cases or censored at the end of the study. Furthermore, the incidence of diabetic nephropathy was determined from the start of type $2 \mathrm{DM}$ diagnosis until the last follow-up visit.

We used the Weibull regression model to identify the predictors of diabetic nephropathy. Person-time at risk was measured starting from the time of initiation of treatment until each patient ended the follow-up. The Schoenfeld residuals test (both global and scaled) and graphical methods were used to check the Cox proportional hazard $(\mathrm{PH})$ assumption. Cox $\mathrm{PH}$ and three parametric models (exponential, Weibull, and log-logistic) were fitted to identify the predictors of diabetic nephropathy. The best model was 
selected by using Akaike information criteria (AIC), Bayesian information criteria (BIC), and log likelihood criteria.

Goodness of fit of the model was assessed by using the Cox-Snell residual technique. Variables with a $p$ value of $\leq 0.2$ were entered into a multivariable model to control the possible effect of confounders. Variables having $p$ value less than 0.05 in the multivariable model were considered significantly associated with the dependent variable. Hazard ratios (HR) with its $95 \%$ confidence interval were computed to determine statistical significance.

2.7. Ethical Consideration. Before the commencement of the study, ethical clearance was obtained from the Institutional Review Board of the University of Gondar. Then, permission letters from officials of University of Gondar Comprehensive Specialized Hospital, Department of Internal Medicine, were processed before data collection. To ensure confidentiality, patient names were not included; instead, code numbers were assigned to depict the results.

\section{Results}

3.1. Baseline Characteristics of Study Participants. Out of the total of 462 newly diagnosed type 2 DM patients, 277 (60\%) were females. The mean duration of diabetes was 8.2 years $(\mathrm{SD}= \pm 3.8)$. More than half, $384(82.9 \%)$ had no history of diabetic retinopathy. About 59 (12.8\%) were on insulin. The mean $( \pm S D)$ age for patients at the start of treatment was $53.2( \pm 10.1)$ years (Table 1$)$.

3.2. Incidence of Diabetic Nephropathy. During the follow up, a total of 63 patients developed diabetic nephropathy. The median time to develop diabetic nephropathy was 94.9 months with an interquartile range (IOR) of 64.1-127.4 months. The overall incidence rate of diabetic nephropathy was 14 (95\% CI 10.8-17.7) cases per 10,000 patient-month with total 45437.1 patient-month observation. Moreover, the proportion of diabetic nephropathy among newly diagnosed T2DM patients was $13.6 \%$.

The cumulative probability of developing diabetic nephropathy among type $2 \mathrm{DM}$ patients who were free from diabetic nephropathy at the start of treatment was 0.0359 at month $40,0.1117$ at month $100,0.3046$ at month 180 , and 0.3973 at month 230 during the follow-up period (Figure 1).

Based on AIC, the Weibull-Cox regression model was the most efficient model to describe the data $(\mathrm{AIC}=416.4)$. According to the Schoenfeld residual global test, the overall full model satisfies the proportional hazard assumption $(X 2=4.67, p<0.912)$. As well, the Cox-Snell residual plot showed the proportional hazard assumption was satisfied (Figure 2).

3.3. Predictors of Diabetic Nephropathy among Type 2 DM Patients. Multivariable analysis result from fitted Weibull regression showed that sex, duration, systolic blood pressure, anemia, and coronary heart disease (CHD) were independent predictors for diabetic nephropathy among type $2 \mathrm{DM}$ patients (Table 2 ).

The risk of developing diabetic nephropathy for patients who have $\mathrm{CHD}$ was 2.69 times higher than that of patients who have no $\mathrm{CHD}(\mathrm{AHR}=2.69,95 \%$ CI 1.42-5.13). The risk of developing diabetic nephropathy was increased by $94 \%$ $(\mathrm{AHR}=1.94,95 \%$ CI $0.97-3.87)$ among newly diagnosed T2DM with anemia than patients with no anemia. The hazard of developing diabetic nephropathy was decreased by $76 \%$ among newly diagnosed type 2 diabetic patients with duration greater than 10 years than patients with duration less than 6 years $(\mathrm{AHR}=0.24,95 \%$ CI $0.11-0.56)$. The risk of developing diabetic nephropathy was decreased by $57 \%$ among female type $2 \mathrm{DM}$ patients than male patients $(\mathrm{AHR}=0.44,95 \%$ CI 0.26-0.73).

\section{Discussion}

This study examined the incidence and predictors of diabetic nephropathy among newly diagnosed T2DM patients at University of Gondar Comprehensive Specialized Hospital. Duration of diabetes, CHD, anemia, and sex were found to be independent predictors of diabetic nephropathy.

The study revealed the incidence rate of diabetic nephropathy was 14 (95\% CI $10.8-17.7)$ cases per 10,000 patient-months of observation. The proportion of diabetic nephropathy was $13.6 \%$ with 14 cases per 10,000 patientmonth observation. The study showed a lower incidence of diabetic nephropathy than studies performed in Ethiopia and United Kingdom (UK) [10, 23]. This inconsistency might be due to the difference in the sample size, diagnostic method, and study design among the studies.

In this study, the median survival time was 94.9 months which is longer than the findings of previous studies performed in Ethiopia, 70.9 months, [10] and Honk Kong, 55.2 months [24]. This could be explained by the increase in access and variety of more potent drugs nowadays than the past periods.

The study identified that among type 2 DM patient's, women had a longer time to develop diabetic nephropathy than men. This result is in line with the studies performed in France [25] and Sweden [26]. This may be explained by the fact that the estrogen hormone plays an important role in protection [27]. The other possible explanation could be that renal function in women is underestimated if creatininebased estimates of the glomerular filtration rate are used despite gender adjustments. In contrast to our results, other studies [23, 24, 28-31] showed that men had a lower risk of developing microvascular complications of DM than women. Therefore, further research is needed to determine whether this gender difference contributes to better outcomes in females with diabetes.

The current study showed that the duration of diabetes was negatively associated with hazard of diabetic nephropathy. This finding is inconsistent with previous studies [24, 27, 32-34]. This might be since our study populations were type $2 \mathrm{DM}$ patients who are more likely to come late to the health facility and seek health services because the disease process of T2DM is more gradual and not as severe 
TABLE 1: Sociodemographic and clinical factors of newly diagnosed T2DM patients in University of Gondar Comprehensive Specialized Hospital, January 2001-February 2016.

\begin{tabular}{|c|c|c|c|}
\hline Variable & Censored (\%) & Diabetic nephropathy (\%) & Total \\
\hline \multicolumn{4}{|l|}{ Sex } \\
\hline Male & $149(37.3)$ & $36(57.1)$ & $185(40.0)$ \\
\hline Female & $250(62.7)$ & $27(42.9)$ & $277(60.0)$ \\
\hline Age & \multicolumn{2}{|c|}{ Mean $=53.2 \pm 10.1$} & \\
\hline$\leq 59$ & $278(69.7)$ & $43(68.3)$ & $321(69.5)$ \\
\hline$\geq 60$ & $121(30.3)$ & $20(31.7)$ & $141(30.5)$ \\
\hline \multicolumn{4}{|l|}{ Diabetic retinopathy } \\
\hline Yes & $68(17.0)$ & $11(17.5)$ & $79(17.1)$ \\
\hline No & $331(83.0)$ & $52(82.5)$ & $383(82.9)$ \\
\hline \multicolumn{4}{|l|}{ Diabetic neuropathy } \\
\hline Yes & $65(16.3)$ & $13(20.6)$ & $78(16.9)$ \\
\hline No & $334(83.7)$ & $50(79.4)$ & $384(83.1)$ \\
\hline \multicolumn{4}{|l|}{ Hypertension } \\
\hline Yes & $89(22.3)$ & $19(30.2)$ & $108(23.4)$ \\
\hline No & $310(77.7)$ & $44(69.8)$ & $354(76.6)$ \\
\hline \multicolumn{4}{|l|}{ Anemia } \\
\hline Yes & $26(6.5)$ & $12(19.1)$ & $38(8.2)$ \\
\hline No & $373(93.5)$ & $51(80.9)$ & $424(91.8)$ \\
\hline \multicolumn{4}{|l|}{ Medication } \\
\hline Dietary modification & $21(5.3)$ & $8(12.7)$ & $29(6.3)$ \\
\hline One oral agent & $261(65.4)$ & $34(54.0)$ & $295(63.8)$ \\
\hline$>1$ oral agent & $70(17.5)$ & $9(14.3)$ & $79(17.1)$ \\
\hline Insulin & $47(11.8)$ & $12(19.1)$ & $59(12.8)$ \\
\hline Duration (year) & \multicolumn{2}{|c|}{ Mean $=8.2 \pm 3.8$} & \\
\hline$<6$ & $100(25.1)$ & $11(17.5)$ & $111(24.0)$ \\
\hline $6-10$ & $199(49.8)$ & $32(50.8)$ & $231(50.0)$ \\
\hline$>10$ & $100(25.1)$ & $20(31.7)$ & $120(26.0)$ \\
\hline FBS & \multicolumn{2}{|c|}{ Mean $=224.6 \pm 80.9$} & \\
\hline$\leq 150$ & $83(20.8)$ & $8(12.7)$ & $91(19.7)$ \\
\hline$>150$ & $316(79.2)$ & $55(87.3)$ & $371(80.3)$ \\
\hline
\end{tabular}

FBS, fasting blood sugar.

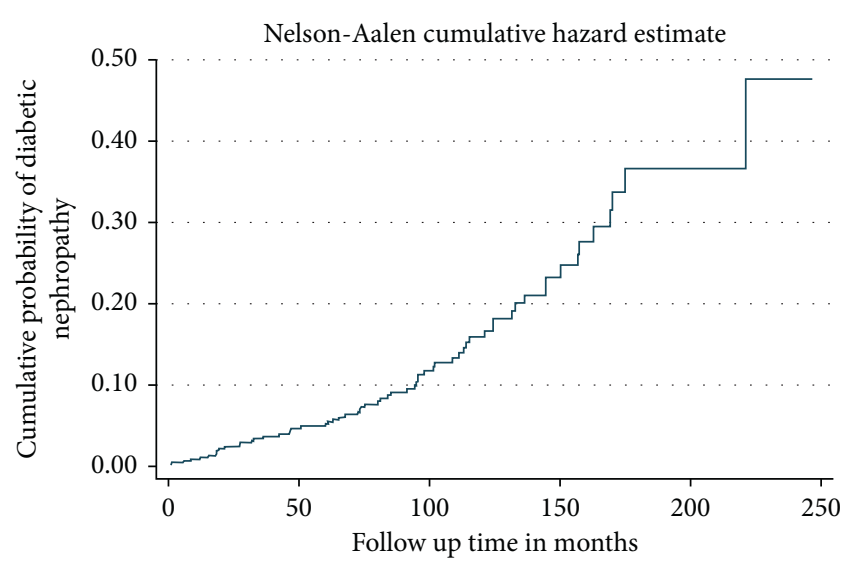

Figure 1: The Nelson-Aalen estimated cumulative curve showing cumulative probability of diabetic nephropathy among type $2 \mathrm{DM}$ patients in University of Gondar Comprehensive Specialized Hospital, January 2001-February 2016.

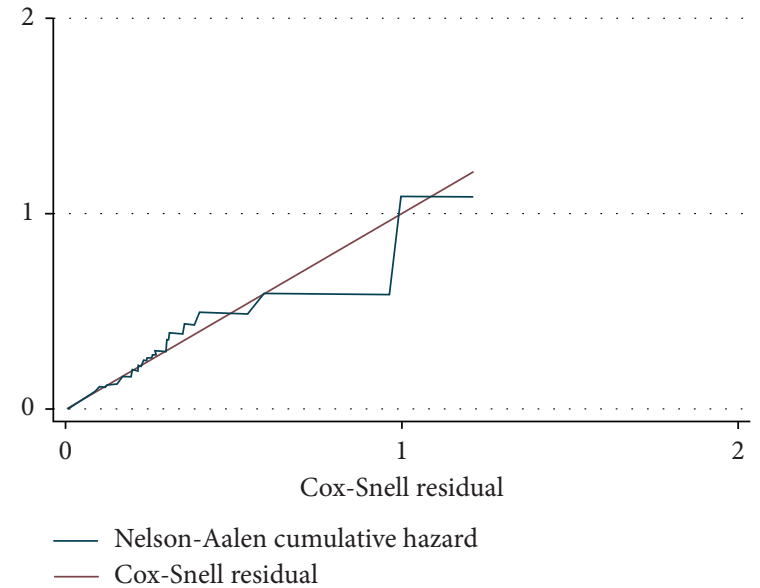

FIgURe 2: Cox-Snell residuals for Weibull PH models of newly diagnosed T2DM patients in University of Gondar Comprehensive Specialized Hospital, January 2001-February 2016. 
TABLE 2: Multivariable analysis using the Weibull regression model for predictors of diabetic nephropathy among type $2 \mathrm{DM}$ patients in University of Gondar Comprehensive Specialized Hospital, January 2001-February 2016.

\begin{tabular}{|c|c|c|}
\hline Variable & Crude HR (95\% CI) & Adjusted HR (95\% CI) \\
\hline \multicolumn{3}{|l|}{ Sex } \\
\hline Male & 1 & 1 \\
\hline Female & $0.44(0.27-0.73)$ & $0.44(0.26-0.73)^{* * *}$ \\
\hline \multicolumn{3}{|l|}{ FBS } \\
\hline$\leq 150$ & 1 & 1 \\
\hline$>150$ & $1.41(0.67-2.96)$ & $1.59(0.75-3.39)$ \\
\hline \multicolumn{3}{|l|}{ Duration } \\
\hline$<6$ years & 1 & 1 \\
\hline $6-10$ years & $0.53(0.26-1.06)$ & $0.50(0.24-1.04)$ \\
\hline$>10$ years & $0.28(0.13-0.62)$ & $0.24(0.11-0.56)^{* * *}$ \\
\hline \multicolumn{3}{|l|}{ SBP } \\
\hline$\leq 140$ & 1 & 1 \\
\hline$>140$ & $1.31(0.75-2.29)$ & $1.91(0.96-3.67)$ \\
\hline \multicolumn{3}{|l|}{ DBP } \\
\hline$\leq 90$ & 1 & 1 \\
\hline$>90$ & $0.67(0.29-1.57)$ & $0.53(0.19-1.41)$ \\
\hline \multicolumn{3}{|l|}{ Anemia } \\
\hline No & 1 & 1 \\
\hline Yes & $2.93(1.56-5.51)$ & $1.94(0.97-3.87)^{*}$ \\
\hline \multicolumn{3}{|c|}{ Diabetic retinopathy } \\
\hline No & 1 & 1 \\
\hline Yes & $0.78(0.41-1.50)$ & $0.71(0.36-1.41)$ \\
\hline Age (year) & $1.02(0.99-1.04)$ & $1.01(0.98-1.03)$ \\
\hline \multicolumn{3}{|l|}{$\mathrm{CHD}$} \\
\hline No & 1 & 1 \\
\hline Yes & $2.91(1.61-5.27)$ & $2.69(1.42-5.13)^{* *}$ \\
\hline
\end{tabular}

as type $1 \mathrm{DM}$ at the early stage of the disease. According to American Diabetes Association medical care standards, diabetes kidney disease may be present at the diagnosis of type 2 diabetes [2]. That is why duration of diabetes and diabetic nephropathy is inversely related in our study. Since the association between duration and diabetic nephropathy is controversial, it needs further research.

We found that CHD is a risk factor for diabetic nephropathy. The finding is in agreement with a previous study [35] which shows that the cell adhesion molecules are raised in both cardiovascular disease and diabetic nephropathy, with levels increasing in stepwise fashion with increasing kidney disease. The possible justification could be that coronary artery stenosis increased renal oxidative stress, fibrosis, inflammation, tubular injury, and microvasculature remodelling [36]. This finding is inconsistent with the study performed in Spain [37]. This is unclear and needs further investigation.

This study found that the presence of anemia was an independent risk factor for diabetic nephropathy. This finding is in line with previous studies performed in USA [11], China [38], and Kenya [39]. This could be linked to chronic hyperglycemia which is involved in the pathogenesis of anemia by causing abnormalities in red blood cells, oxidative stress, autonomic neuropathy, and renal sympathetic denervation. These conditions put the renal interstitium in a hypoxic state, and consequently, the production of erythropoietin by peritubular fibroblasts is impaired.

The strength of this study was following patients for long duration. This study is not free from limitation. Since the data were secondary data, some potentially important predictors were not available like smoking behavior, family history of diabetes, and kidney disease. Furthermore, use of secondary data collected retrospectively results in incompleteness.

\section{Conclusion}

In this retrospective follow-up study, findings showed that the incidence of diabetic nephropathy among type 2 diabetes mellitus patients remains a significant public health problem. Duration of diabetes $>10$ years and female sex reduced the risk of diabetic nephropathy. Besides this, coronary heart disease and anemia increased the risk diabetic nephropathy among type 2 diabetes mellitus patients. In light of these findings, health professionals in the DM follow-up clinics should give targeted intervention for type $2 \mathrm{DM}$ patients with coronary heart disease comorbidity and anemia. Early screening and treatment of diabetes complications would be an essential part of DM care, delaying the onset of diabetic nephropathy.

\section{Abbreviations}

AHR: Adjusted hazard ratio

AIC: Akaike's information criterion

BIC: Bayesian information criterion

CHD: Coronary heart disease

CHR: Crude hazard ratio

DM: Diabetes mellitus

ESRD: End-stage renal disease

HR: Hazard ratio

PH: Proportional hazard

T2DM: Type 2 diabetes mellitus.

\section{Data Availability}

The data used to support the findings of this study are included within the article.

\section{Consent}

Informed consent is not required as the whole data had been retrieved from the medical records of patient.

\section{Conflicts of Interest}

The authors declare that they have no conflicts of interest.

\section{Authors' Contributions}

SA involved in conception of the work, design of the work, acquisition of data, analysis, and interpretation of data. SA, $\mathrm{BS}, \mathrm{AB}, \mathrm{ZT}$, and TA involved in data curation, drafting the 
article, and revising it critically for intellectual content, validation, and final approval of the version to be published. All authors read and approved the final manuscript.

\section{Acknowledgments}

The authors express their appreciation to University of Gondar Comprehensive Specialized Hospital, particularly the DM clinic staff for their kind cooperation during data collection. The authors are also grateful to the data collectors.

\section{References}

[1] G. Roglic, "WHO global report on diabetes: a summary," International Journal of Noncommunicable Diseases, vol. 1, no. 1, p. 3, 2016.

[2] Association, A. D., "Standards of medical care in diabetes2017 Abridged for primary care providers," Clinical Diabetes, vol. 35, no. 1, pp. 5-26, 2017.

[3] Association, A. H., Kidney Disease and Diabetes, Association, A. H., Mount Laurel, NJ, USA, 2016.

[4] R. C. Atkins and P. Zimmet, "Diabetic kidney disease: act now or pay later," Nephrology Dialysis Transplantation, vol. 25, no. 2, pp. 331-333, 2010.

[5] G. Wang, J. Ouyang, S. Li et al., "The analysis of risk factors for diabetic nephropathy progression and the construction of a prognostic database for chronic kidney diseases," Journal of Translational Medicine, vol. 17, no. 1, pp. 264-312, 2019.

[6] Federation, I.D., IDF Diabetes Atlas, Federation, I.D., Tel Aviv, Israel, 9th edition, 2019.

[7] N. G. Forouhi and N. J. Wareham, "Epidemiology of diabetes," Medicine, vol. 38, no. 11, pp. 602-606, 2010.

[8] Y. Zheng, S. H. Ley, and F. B. Hu, "Global aetiology and epidemiology of type 2 diabetes mellitus and its complications," Nature Reviews Endocrinology, vol. 14, no. 2, pp. 88-98, 2018.

[9] J. J. N. Noubiap, J. Naidoo, and A. P. Kengne, "Diabetic nephropathy in Africa: a systematic review," World Journal of Diabetes, vol. 6, no. 5, p. 759, 2015.

[10] A. H. Geletu, A. S. Teferra, M. M. Sisay, and D. F. Teshome, "Incidence and predictors of chronic kidney diseases among type 2 diabetes mellitus patients at St. Paul's hospital, Addis Ababa, Ethiopia," BMC Research Notes, vol. 11, no. 1, p. 532, 2018.

[11] M. Gaballa and Y. Farag, "Predictors of diabetic nephropathy," Open Medicine, vol. 8, no. 3, pp. 287-296, 2013.

[12] V. Vimalkumar, C. An, and S. Padmanaban, "Prevalence and risk factors of nephropathy in type 2 diabetic patients," International Journal of Collaborative Research on Internal Medicine \& Public Health, vol. 3, no. 8, 2011.

[13] W. J. Lee, L. Sobrin, M. J. Lee, M. H. Kang, M. Seong, and H. Cho, "The relationship between diabetic retinopathy and diabetic nephropathy in a population-based study in Korea (KNHANES V-2, 3)," Investigative Opthalmology \& Visual Science, vol. 55, no. 10, pp. 6547-6553, 2014.

[14] M. H. Ahmed, E. S. Elwali, H. Awadalla, and A. O. Almobarak, "The relationship between diabetic retinopathy and nephropathy in Sudanese adult with diabetes: population based study," Diabetes \& Metabolic Syndrome: Clinical Research \& Reviews, vol. 11, pp. S333-S336, 2017.

[15] J. Zhang, Y. Wang, L. Li et al., "Diabetic retinopathy may predict the renal outcomes of patients with diabetic nephropathy," Renal Failure, vol. 40, no. 1, pp. 243-251, 2018.
[16] Organization W.H., Guidelines for the Prevention, Management and Care of Diabetes Mellitus, Organization W.H., Geneva, Switzerland, 2006.

[17] A. D. Deshpande, M. Harris-Hayes, and M. Schootman, "Epidemiology of diabetes and diabetes-related complications," Physical Therapy, vol. 88, no. 11, pp. 1254-1264, 2008.

[18] Federation I.D., IDF Diabetes Atlas, Federation I.D., Tel Aviv, Israel, Eighth edition, 2017.

[19] J. E. Bennett, G. A. Stevens, C. D. Mathers et al., "NCD countdown 2030: worldwide trends in non-communicable disease mortality and progress towards sustainable development goal target 3.4," The Lancet, vol. 392, no. 10152, pp. 1072-1088, 2018.

[20] J. Morton, S. Zoungas, Q. Li et al., "Low HDL cholesterol and the risk of diabetic nephropathy and retinopathy: results of the advance study," Diabetes Care, vol. 35, no. 11, pp. 2201-2206, 2012.

[21] D. W. Cockcroft and H. Gault, "Prediction of creatinine clearance from serum creatinine," Nephron, vol. 16, no. 1, pp. 31-41, 1976.

[22] F. Persson and P. Rossing, "Diagnosis of diabetic kidney disease: state of the art and future perspective," Kidney International Supplements, vol. 8, no. 1, pp. 2-7, 2018.

[23] R. Retnakaran, C. A. Cull, K. I. Thorne, A. I. Adler, and R. R. Holman, "Risk factors for renal dysfunction in type 2 diabetes: U.K. prospective diabetes study 74," Diabetes, vol. 55, no. 6, pp. 1832-1839, 2006.

[24] A. O. Y. Luk, W.-Y. So, R. C. W. Ma et al., "Metabolic syndrome predicts new onset of chronic kidney disease in 5,829 patients with type 2 diabetes: a 5 -year prospective analysis of the Hong Kong diabetes registry," Diabetes Care, vol. 31, no. 12, pp. 2357-2361, 2008.

[25] A. De Hauteclocque, S. Ragot, Y. Slaoui et al., "The influence of sex on renal function decline in people with type 2 diabetes," Diabetic Medicine, vol. 31, no. 9, pp. 1121-1128, 2014.

[26] H. Afghahi, J. Cederholm, B. Eliasson et al., "Risk factors for the development of albuminuria and renal impairment in type 2 diabetes--the Swedish national diabetes register (NDR)," Nephrology Dialysis Transplantation, vol. 26, no. 4, pp. 1236-1243, 2011.

[27] R. Unnikrishnan, M. Rema, R. Pradeepa et al., "Prevalence and risk factors of diabetic nephropathy in an urban South Indian population: the Chennai urban rural epidemiology study (CURES 45)," Diabetes Care, vol. 30, no. 8, pp. 2019-2024, 2007.

[28] A. Kajiwara, A. Kita, J. Saruwatari et al., "Sex differences in the renal function decline of patients with type 2 diabetes," Journal of diabetes research, vol. 2016, Article ID 4626382, 2016.

[29] H. F. Wolde, A. Atsedeweyen, A. Jember et al., "Predictors of vascular complications among type 2 diabetes mellitus patients at University of Gondar referral hospital: a retrospective follow-up study," BMC Endocrine Disorders, vol. 18, no. 1, p. 52, 2018.

[30] M. K. Yu, W. Katon, and B. A. Young, "Associations between sex and incident chronic kidney disease in a prospective diabetic cohort," Nephrology, vol. 20, no. 7, pp. 451-458, 2015.

[31] R. Raman, A. Gupta, S. Krishna, V. Kulothungan, and T. Sharma, "Prevalence and risk factors for diabetic microvascular complications in newly diagnosed type II diabetes mellitus. Sankara Nethralaya diabetic retinopathy epidemiology and molecular genetic study (SN-DREAMS, report 27)," Journal of Diabetes and its Complications, vol. 26, no. 2, pp. 123-128, 2012. 
[32] K. Al-Rubeaan, A. M. Youssef, S. N. Subhani et al., "Diabetic nephropathy and its risk factors in a society with a type 2 diabetes epidemic: a Saudi national diabetes registry-based study," PLoS One, vol. 9, no. 2, 2014.

[33] S. Damtie, B Biadgo, H. W Baynes et al., "Chronic kidney disease and associated risk factors assessment among diabetes mellitus patients at a tertiary hospital, Northwest Ethiopia," Ethiopian journal of health sciences, vol. 28, no. 6, pp. 691-700, 2018.

[34] A. H. Alrawahi, S. G. A. Rizvi, D. Al-Riyami, and Z. AlAnqoodi, "Prevalence and risk factors of diabetic nephropathy in omani type 2 diabetics in Al-dakhiliyah region," Oman Medical Journal, vol. 27, no. 3, pp. 212-216, 2012.

[35] T. Wu, K. C. McGrath, and A. K. Death, "Cardiovascular disease in diabetic nephropathy patients: cell adhesion molecules as potential markers?" Vascular Health and Risk Management, vol. 1, no. 4, pp. 309-316, 2005.

[36] D. Sun, A. Eirin, X.-Y. Zhu et al., "Experimental coronary artery stenosis accelerates kidney damage in renovascular hypertensive swine," Kidney International, vol. 87, no. 4, pp. 719-727, 2015.

[37] M. A. Salinero-Fort, F. J. San Andrés-Rebollo, C. De BurgosLunar et al., "Five-year incidence of chronic kidney disease (stage 3-5) and associated risk factors in a Spanish cohort: the MADIABETES study," PLoS One, vol. 10, no. 4, 2015.

[38] K. Guo, L. Zhang, F. Zhao et al., "Prevalence of chronic kidney disease and associated factors in Chinese individuals with type 2 diabetes: cross-sectional study," Journal of Diabetes and its Complications, vol. 30, no. 5, pp. 803-810, 2016.

[39] V. Mwenda, J. Githuku, G. Gathecha, B. M. Wambugu, Z. G. Roka, and W. O. Ong'or, "Prevalence and factors associated with chronic kidney disease among medical inpatients at the Kenyatta national hospital, Kenya, 2018: a crosssectional study," The Pan African Medical Journal, vol. 33, p. 321, 2019. 